\title{
Visibility and Origin of Compact Interplanetary Radio Type IV Bursts
}

\author{
Nasrin Talebpour Sheshvan ${ }^{1}$ (D) $\cdot$ Silja Pohjolainen ${ }^{1,2}$
}

Received: 9 May 2018 / Accepted: 19 October 2018 / Published online: 30 October 2018

(C) The Author(s) 2018

\begin{abstract}
We have analyzed radio type IV bursts in the interplanetary (IP) space at decameter-hectometer (DH) wavelengths to determine their source origin and a reason for the observed directivity. We used radio dynamic spectra from the instruments on three different spacecraft, STEREO-A, Wind, and STEREO-B, which were located approximately 90 degrees apart from each other in 2011-2012, and thus gave a 360 degree view of the Sun. The radio data were compared to white-light and extreme ultraviolet (EUV) observations of flares, EUV waves, and coronal mass ejections (CMEs) in five solar events. We find that the reason that compact and intense DH type IV burst emission is observed from only one spacecraft at a time is the absorption of emission in one direction and that the emission is blocked by the solar disk and dense corona in the other direction. The geometry also makes it possible to observe metric type IV bursts in the low corona from a direction where the higher-located DH type IV emission is not detectable. In the absorbed direction we found streamers, and they were estimated to be the locations of type II bursts, caused by shocks at the CME flanks. The high-density plasma was therefore most probably formed by shockstreamer interaction. In some cases, the type II-emitting region was also capable of stopping later-accelerated electron beams, which were visible as type III bursts that ended near the type II burst lanes.
\end{abstract}

Keywords Coronal mass ejections · Initiation and propagation · Radio bursts · Meter-wavelengths and longer ( $\mathrm{m}, \mathrm{dkm}, \mathrm{hm}, \mathrm{km}) \cdot$ Type II · Type IV

N. Talebpour Sheshvan natash@utu.fi

S. Pohjolainen silpoh@utu.fi

1 Department of Physics and Astronomy, University of Turku, Turku, Finland

2 Tuorla Observatory, Department of Physics and Astronomy, University of Turku, Turku, Finland 


\section{Introduction}

Coronal mass ejections (CMEs) are large-scale phenomena of plasma and magnetic field eruption from the Sun into the interplanetary (IP) medium. Solar events such as flares and CMEs accelerate particles with different mechanisms, and cause them to propagate from the solar corona into the IP space. For reviews see, e.g., Pick and Vilmer (2008) and Nindos et al. (2008). The particle paths and their directions and locations can be studied using radio bursts at a wide wavelength range, from metric to kilometric wavelengths.

Solar radio bursts in the plasma regime (below $\approx 1 \mathrm{GHz}$ ) are generally classified into different types based on their appearance in the dynamic spectrum and their emission mechanism. The most common types are radio type II and type III bursts. Type II bursts are thought to be due to propagating shock fronts that accelerate electrons, and type III bursts are caused by propagating electron beams. Both types of emission are produced when suprathermal electrons interact with the surrounding plasma. This creates radio emission at the local plasma frequency $f_{\mathrm{p}}$ and/or its harmonics. The particles in type III bursts are typically flare-accelerated electrons traveling along coronal magnetic field lines (Krupar et al., 2018), while type II bursts have been associated with CME-induced shock waves (Cane, 1984; Leblanc et al., 2001) .

The more rare radio type IV bursts have been closely associated with expanding and/or rising magnetized plasma structures. The emission mechanism can therefore be both synchrotron emission by trapped high-energy electrons gyrating in the magnetic field and plasma emission as the magnetic cloud lifts off from the Sun. Typically, metric type IV bursts are divided into two categories, stationary and moving. The characteristics of stationary type IV bursts have been defined by Kundu (1965): they are located near a flare, are strongly circularly polarized, smooth and broad band, and are located low in the corona near the corresponding plasma level. The stationary type IV bursts show no systematic movement, and the bursts may also occur without any associated type II emission. It has been suggested that the source of emission is particles accelerated in a flare that are trapped in post-flare loops and arcades. Moving type IV bursts, on the other hand, show definite frequency drifts toward the lower frequencies, and they are mostly thought to represent particles that are trapped in rising CME structures. Kundu (1965) also stated that type IV bursts show directivity in their emission. Some type IV bursts extend in the dynamic spectra to decameter-hectometer $(\mathrm{DH})$ wavelengths, and they can be recorded by space-borne instruments at frequencies below $\approx 20 \mathrm{MHz}$ (Earth ionosphere limit). The characteristics of IP moving type IV bursts have recently been listed and studied by Hillaris, Bouratzis, and Nindos (2016), and their catalog contains bursts recorded in 1998-2012 by the radio experiment on board the Wind satellite. Directivity in IP type IV burst emission was later noted by Gopalswamy et al. (2016).

Closely associated with high-speed CMEs are EUV-waves, observed in the extreme ultraviolet (EUV) wavelength range in the low corona. These bright features and the dimming behind them typically start from the flare location and propagate globally over the solar disk. However, no strong correlation was found between the global EUV disturbance speeds and flare intensities, or CME magnitudes (Nitta et al., 2013). Recently, Kwon and Vourlidas (2017) found that halo-CME widths are in agreement with the widths of EUV waves in the low corona, suggesting a common origin for these structures. In some cases, the heights of EUV waves have been found to be compatible with the heights of radio type II burst sources (Cunha-Silva, Fernandes, and Selhorst, 2015). Since radio type IV bursts can be observed over a wide frequency range, the result of the studies made over the past decades has been to define type IV bursts as any broad-band, long-duration, continuum-like emission occurring 
after a flare. Moreover, imaging has only been available for decimetric-metric bursts that occur in the low corona, but not for DH type IV bursts in the IP space.

In 2007, two more spacecraft with a radio spectrograph were launched to orbit the Sun, STEREO A and B, which together with the instrument onboard Wind, made a 3D view of the Sun possible, if not real imaging. In this study we use the radio data from these three spacecraft, and we try to determine where IP type IV bursts are located, whether there is directivity in their emission, and if they are a continuation of metric type IV burst emission.

\section{Data Analysis}

The time period to find DH type IV bursts was set to 2011-2012 in order to have a full 3D view of the Sun, as then the spacecraft were orbiting the Sun with an $\approx 90^{\circ}$ angular separation, and the far side of the Sun could be imaged in EUV and white-light with the instruments on board STEREO A and B, see Figure 1. The Earth-side imaging was provided by the Solar Dynamic Observatory (SDO) and the Solar and Heliospheric Observatory (SOHO), and magnetograms by the Helioseismic and Magnetic Imager (HMI) onboard SDO.

The DH type IV bursts were selected from the catalog of solar type II and type IV bursts detected by the WAVES instruments on Wind (Bougeret et al., 1995) and STEREO A and B (Bougeret et al., 2008). The catalog has been prepared by Michael L. Kaiser. Altogether, $11 \mathrm{DH}$ type IV events were found from this time period, but only 5 of them were found to show a compact, intense, and long-duration DH type IV burst, with radio and imaging observations from all the three viewing angles. These 5 events and their sources are listed in Tables 1 and 2. Figure 1 shows the spacecraft positions relative to the Sun on the first and last event date.

The highest observing frequencies for Wind/WAVES and STEREO/WAVES are $14 \mathrm{MHz}$ and $16 \mathrm{MHz}$, respectively. In the case of plasma emission, these frequencies correspond to densities of $2.4 \times 10^{6} \mathrm{~cm}^{-3}$ and $3.2 \times 10^{6} \mathrm{~cm}^{-3}$ (plasma emission frequency $f_{\mathrm{p}} \approx 9000 \sqrt{n_{\mathrm{e}}}$, where $f_{\mathrm{p}}$ is in $\mathrm{Hz}$ and $n_{\mathrm{e}}$ in $\mathrm{cm}^{-3}$ ).

Three of the DH type IV bursts were preceded by metric type IV bursts that were observed with ground-based instruments from Earth. The other two DH type IVs had their
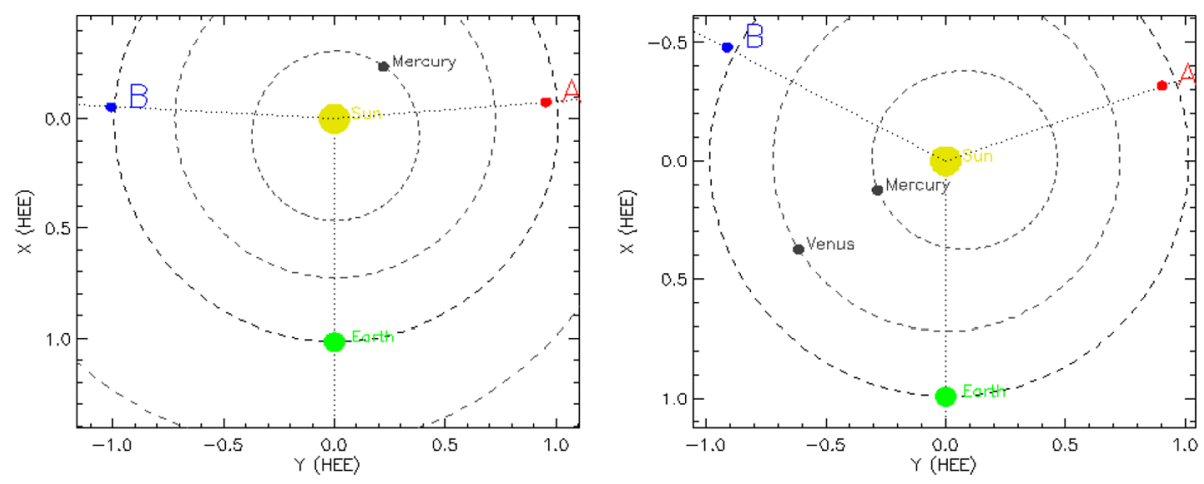

Figure 1 Locations of the STEREO A (red circle) and B (blue circle) spacecraft relative to the Earth (green circle) on 4 June 2011 (left) and 5 March 2012 (right). These are the first and last event dates in our sample. The plots show the increasing orbital distance with respect to Earth and the Wind satellite located near L1. During this time period, the Sun could be viewed in 3D (https://stereo-ssc.nascom.nasa.gov). 
Table 1 DH type IV bursts observed from three different viewing angles.

\begin{tabular}{lllll}
\hline $\begin{array}{l}\text { Date } \\
\text { yyyy-mm-dd }\end{array}$ & $\begin{array}{l}\text { Type IV appearance } \\
\text { UT }\end{array}$ & $\begin{array}{l}\text { STEREO-B } \\
\text { (source loc.) }\end{array}$ & $\begin{array}{l}\text { Type IV Wind } \\
\text { (source loc.) }\end{array}$ & $\begin{array}{l}\text { STEREO-A } \\
\text { (source loc.) }\end{array}$ \\
\hline $2011-06-04$ & $07: 12-09: 00$ & - & - & strong (W50) \\
$2011-06-04$ & $22: 15-23: 30^{2}$ & - & - & strong (W70) \\
$2011-09-22$ & $11: 10-12: 40$ & strong (W10) & - & - \\
$2012-01-27$ & $18: 30-20: 10$ & $-(\mathrm{N}-\operatorname{limb})$ & - & strong (E50) \\
$2012-03-05$ & $04: 15-06: 00$ & faint (W80) & strong (E50) & - \\
\hline
\end{tabular}

${ }^{1}$ Time of IP type IV start and end at $16-14 \mathrm{MHz}$ (instrument limit).

${ }^{2}$ First part, a second more narrow-band enhancement follows at 23:45-01:20 UT.

Table 2 Flares and metric type IV bursts prior to the DH type IV bursts.

\begin{tabular}{llllll}
\hline $\begin{array}{l}\text { Date } \\
\text { yyyy-mm-dd }\end{array}$ & $\begin{array}{l}\text { Flare start } \\
\text { UT }\end{array}$ & $\begin{array}{l}\text { Flare maximum } \\
\text { UT }\end{array}$ & GOES class & $\begin{array}{l}\text { Flare location } \\
\text { (Earth view) }\end{array}$ & $\begin{array}{l}\text { Metric } \\
\text { type IV }\end{array}$ \\
\hline $2011-06-04$ & $06: 20$ & & & N20W140 & not available \\
$2011-06-04$ & $21: 45$ & & N20W150 & not available \\
$2011-09-22$ & $10: 29$ & $11: 01$ & X1.4 & N13E78 & $10: 40-13: 40$ \\
$2012-01-27$ & $17: 37$ & $18: 37$ & X1.7 & N27W71 & $18: 15-18: 35$ \\
$2012-03-05$ & $02: 30$ & $04: 09$ & $X 1.1$ & N17E52 & $04: 20-05: 20$ \\
\hline
\end{tabular}

Table 3 EUV waves associated with the DH type IV events.

\begin{tabular}{|c|c|c|c|c|}
\hline $\begin{array}{l}\text { Date } \\
\text { yyyy-mm-dd }\end{array}$ & $\begin{array}{l}\text { EUV wave }{ }^{1} \\
\mathrm{~km} \mathrm{~s}^{-1}\end{array}$ & $\begin{array}{l}\text { STEREO-B/EUVI } \\
\text { (WAVES) }\end{array}$ & $\begin{array}{l}\text { EUV wave (radio) } \\
\text { SDO/AIA } \\
\text { (Wind/WAVES) }\end{array}$ & $\begin{array}{l}\text { STEREO-A/EUVI } \\
\text { (WAVES) }\end{array}$ \\
\hline 2011-06-04 & & - (no IV) & - (no IV) & global (strong IV) \\
\hline 2011-06-04 & & at limb (no IV) & - (no IV) & global (strong IV) \\
\hline 2011-09-22 & 595 & global (strong IV) & at limb (no IV) & $-($ no IV $)$ \\
\hline $2012-01-27$ & 635 & N-quarter (no IV) & W-quarter (no IV) & global (strong IV) \\
\hline $2012-03-05$ & 915 & half-disk (faint IV) & global (strong IV) & $-($ no IV $)$ \\
\hline
\end{tabular}

${ }^{1}$ Large-scale coronal propagating fronts observed by SDO/AIA (Nitta et al., 2013); note that the velocity value comes from Earth-view observations only.

origins on the far side of the Sun, and therefore the radio emission at lower atmospheric heights could not be observed (Table 2). Two of the five flares originated from the backside of the Sun, with no X-ray observations, and therefore their GOES X-ray flare class is not known. The other three were GOES X-class flares.

All the type IV events were associated with EUV waves, and they are listed in Table 3. All the observed strong, long-duration type IV bursts were associated with global EUV waves that were observed to cross the solar disk in this viewing angle. The EUV waves that were visible only near the limb, or which covered only a small portion of the solar disk, had either no corresponding type IV emission or the type IV was faint in intensity and ended earlier.

The associated CMEs and type II shocks are listed in Table 4. All the CMEs had very high speed, in the range of $1600-2900 \mathrm{~km} \mathrm{~s}^{-1}$ near the time of type IV burst appearance 
Table 4 CMEs and type II bursts associated with the DH type IV events.

\begin{tabular}{llllllll}
\hline $\begin{array}{l}\text { Date } \\
\text { yyyy-mm-dd }\end{array}$ & $\begin{array}{l}\text { CME } \\
\text { height } \\
\mathrm{R}_{\odot}{ }^{1}\end{array}$ & $\begin{array}{l}\text { CME } \\
\text { speed }^{2} \\
\mathrm{~km} \mathrm{~s}^{-1}\end{array}$ & $\begin{array}{l}\text { Type IV } \\
\text { lowest } \\
\mathrm{UT}\end{array}$ & $\begin{array}{l}\text { Type IV } \\
\text { lowest } \\
\mathrm{MHz}\end{array}$ & $\begin{array}{l}\text { Type II } \\
\text { freq. } \\
\mathrm{MHz}\end{array}$ & $\begin{array}{l}\text { Type II } \\
\text { height }^{4} \\
\mathrm{R}_{\odot}\end{array}$ & $\begin{array}{l}\text { CME } \\
\text { height }^{5} \\
\mathrm{R}_{\odot}\end{array}$ \\
\hline $2011-06-04$ & 6.6 & 1700 & $08: 00$ & 6 & 1.6 & 8.0 & 12.8 \\
$2011-06-04$ & 6.3 & 2900 & $23: 05$ & 6 & 0.8 & 11.7 & 17.8 \\
$2011-09-22$ & 8.4 & 2200 & $12: 15$ & 8 & 0.7 & 12.6 & 18.5 \\
$2012-01-27$ & 3.8 & 2000 & $19: 30$ & 9 & 1.4 & 8.3 & 16.1 \\
$2012-03-05$ & 6.6 & 1600 & $05: 15$ & 7 & 1.0 & 10.4 & 14.8 \\
\hline
\end{tabular}

${ }^{1} \mathrm{CME}$ height at the time of the type IV appearance, interpolated or extrapolated when not observed at that time. The type IV appearance at $16-14 \mathrm{MHz}$ (instrument limit) corresponds to a height of $2-3 \mathrm{R}_{\odot}$, depending on the atmospheric density model.

${ }^{2} \mathrm{CME}$ speed from a second-order fit to the observed plan-of-sky heights near the time of type IV burst appearance.

${ }^{3}$ Type II burst frequency at the time of lowest type IV frequency.

${ }^{4}$ Type II burst height from the density model of Vršnak, Magdalenič, and Zlobec (2004).

${ }^{5} \mathrm{CME}$ height at the time of the lowest type IV frequency, interpolated or extrapolated when not observed at that time.

(second-order fit to the observed plane-of-sky heights, from the LASCO CME catalog). The CME on 27 January 2012 accelerated while all the other CMEs decelerated.

The lowest type IV frequencies mark the time when the particle supply ends and/or the trapping loops start to shrink. The lowest observed type IV frequencies were $9-6 \mathrm{MHz}$, which correspond to heliocentric heights of 3.4-4.1 $\mathrm{R}_{\odot}$ when calculated using the atmospheric density model of Vršnak, Magdalenič, and Zlobec (2004). The type II bursts were at that time already at much lower frequencies, at $1.6-0.7 \mathrm{MHz}$, which correspond to heights of 8.0-12.6 $\mathrm{R}_{\odot}$. The height separation between the front of the type IV emission source and the propagating type II shock was therefore in the range of $4-9 R_{\odot}$.

When we compare the type II heights with the simultaneous CME leading front heights, it is evident that the type II bursts were not due to CME bow shocks, but were shocks at the CME flanks (height separation of about $4-8 \mathrm{R}_{\odot}$ ).

The magnetic field structure near the eruption location on each day is shown in Figure 2. Figures $3-7$ present the radio dynamic spectra and the corresponding coronagraph and EUV difference images for the five events, with the three different views from the three spacecraft.

\section{Results}

\subsection{First Event on 4 June 2011}

The flare started approximately at 06:20 UT from the far side of the Sun, near N20W140. The halo CME was first observed by SOHO/LASCO at 06:48 UT at height $2.58 \mathrm{R}_{\odot}$, propagating to north-west with a linear fit speed of $1407 \mathrm{~km} \mathrm{~s}^{-1}$ (LASCO CME Catalog). An EUV wave occurred on the backside, observed by STEREO-A/EUVI, and it originated from the active region located at $\sim \mathrm{W} 50$ in this field of view. The first brightening phase of the EUV wave started at 06:30 UT, after which a dimming phase was observed at 06:50 UT. The 

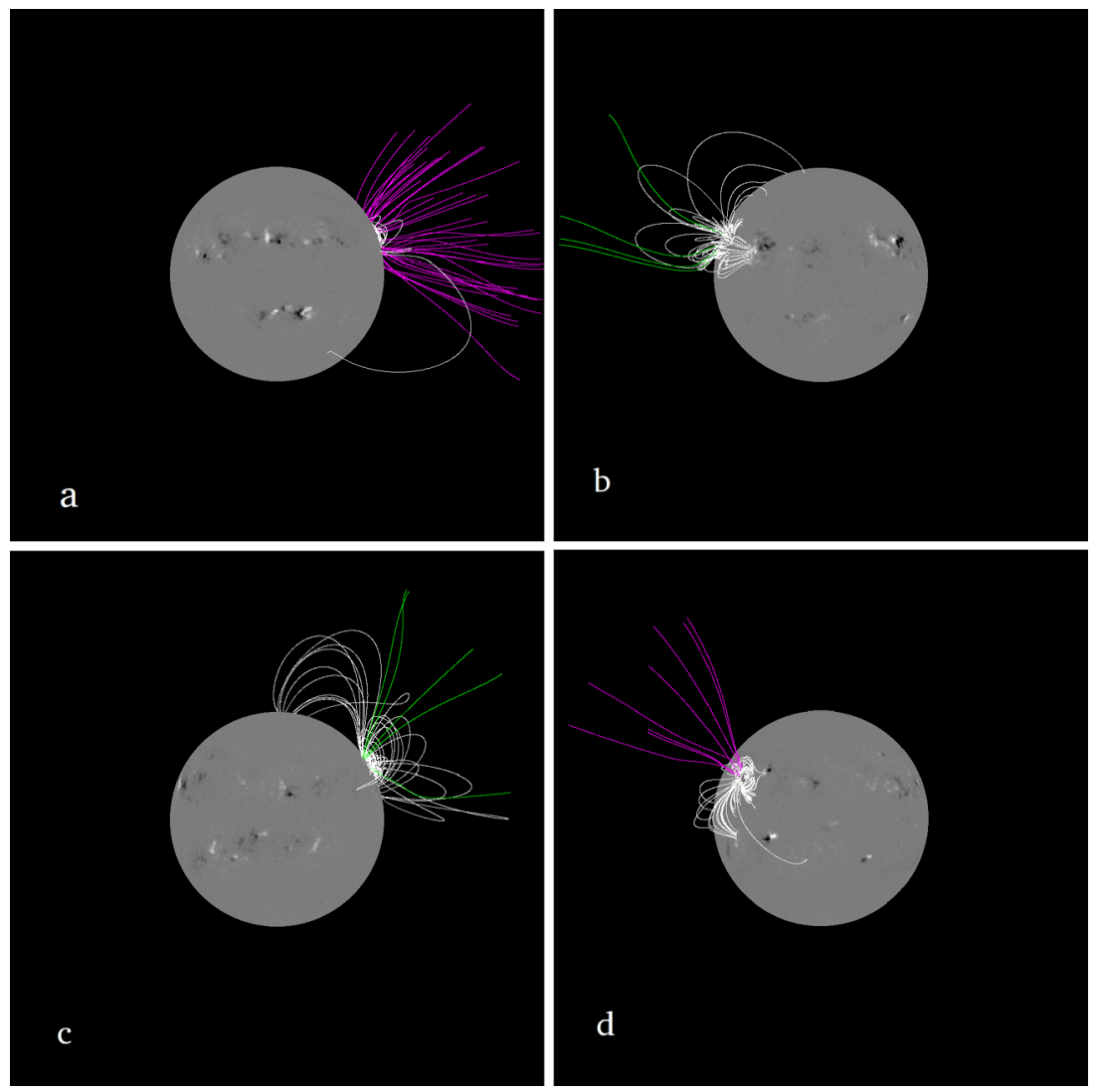

Figure 2 Potential field source surface (PFSS) maps made from the SDO/HMI magnetograms (Earth view) from L1 provide an approximation of the magnetic field line structures in each event, on (a) 4 June 2011, (b) 22 September 2011, (c) 27 January 2012, and (d) 5 March 2012. In the above maps, the purple and green lines are open field lines indicating negative and positive polarity, respectively. The white lines are closed field lines. Open field lines show locations where accelerated particles can escape from the active region, e.g., electron beams observed as type III bursts.

EUV wave crossed most part of the disk within the STEREO-A view, but the wave was not observed on the disk in the other fields-of-view.

The DH type IV event became visible in the dynamic spectrum at 07:12 UT at $16 \mathrm{MHz}$, and it was observed by STEREO-A but not by Wind or STEREO-B. At 08:00 UT it reached the lowest frequency of about $6 \mathrm{MHz}$. As the flare site was at $\sim \mathrm{W} 50$ in STEREO-A view we cannot know if a metric type IV burst existed, as radio emission originating from low heights would have been blocked by the solar disk toward the Earth.

The type IV burst was atypical in the sense that it showed type III-like burst structures superposed, and started from the wide-band emission (Figure 3, top right dynamic spectrum). This indicates that several open field lines existed along which particles could stream and/or escape from the trap, see the potential magnetic field lines plotted in Figure 2a.

A type II burst was observed by STEREO-A and Wind, but not by STEREO-B. As the most probable location for the type II burst source is on the western flank of the CME (Earth view, Figure 3 middle left difference image), it would have been behind the Sun in the STEREO-B view and hence undetectable. 

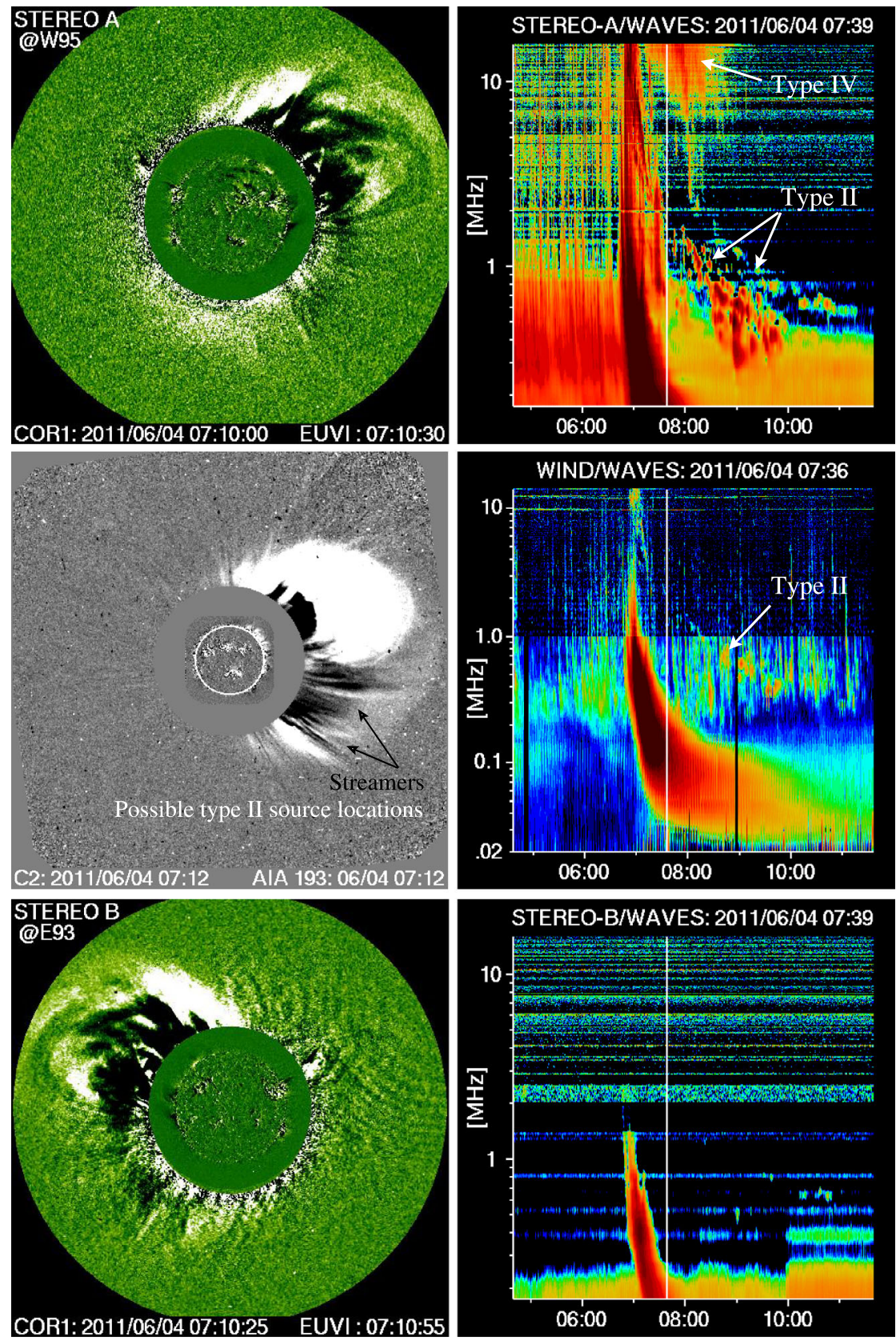

Figure 3 Solar event on 4 June 2011: Flare location on the far side of the Sun, near N20W140 (Earth view).

\subsection{Second Event on 4 June 2011}

The next type IV event occurred on the same day, 15 hours later, on 4 June 2011. The flare originated from the same active region on the far side of the Sun, starting at 21:45 UT 

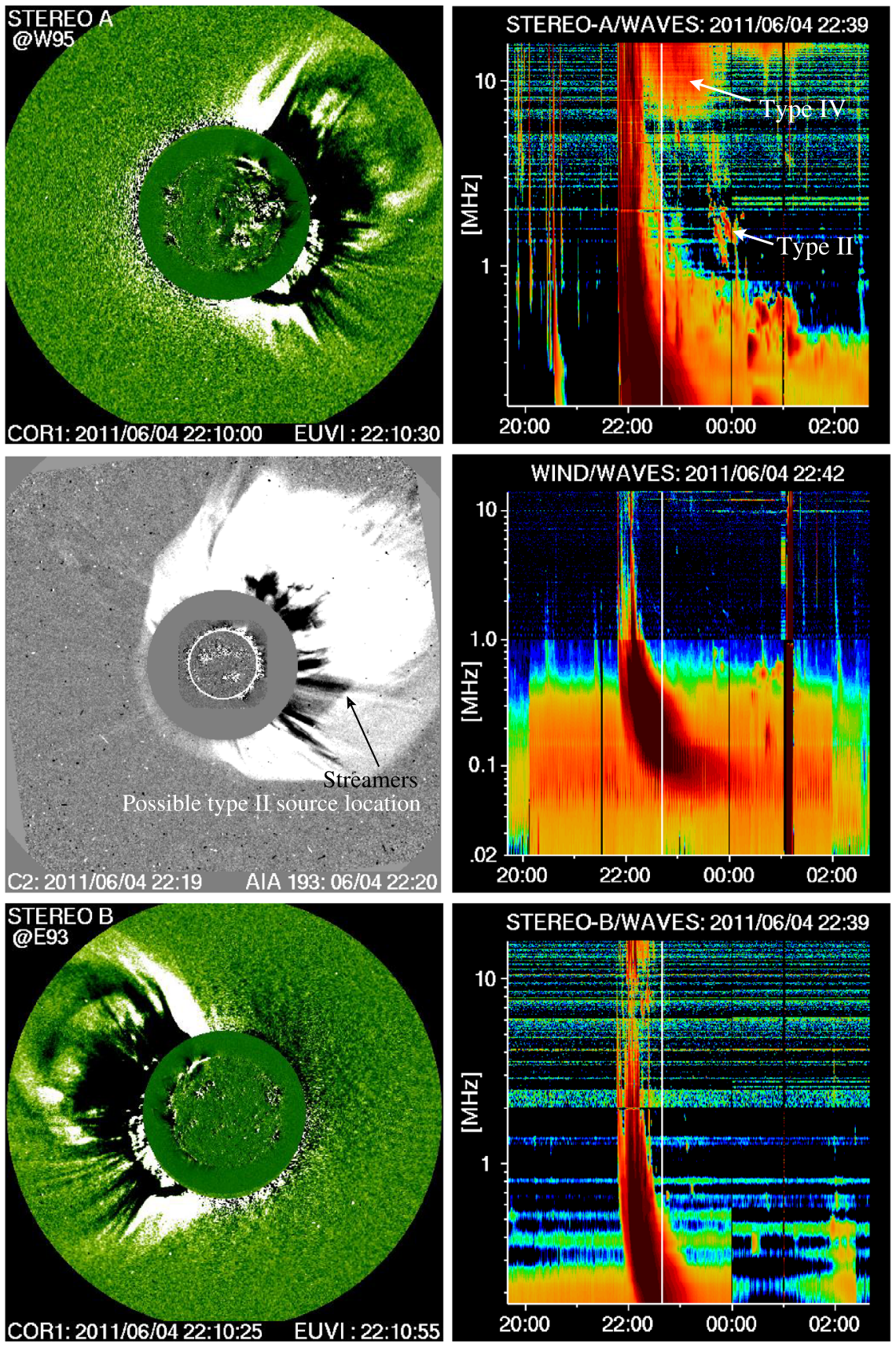

Figure 4 Solar event on 4 June 2011: Flare location on the far side of the Sun, near N20W150 (Earth view).

and located approximately at N20W150 ( W60 in STEREO-A view). The magnetic field structures may have been the same as in the earlier event on the same day (Figure 2a), but as the active region had now rotated well behind the limb, we cannot be sure of the actual 

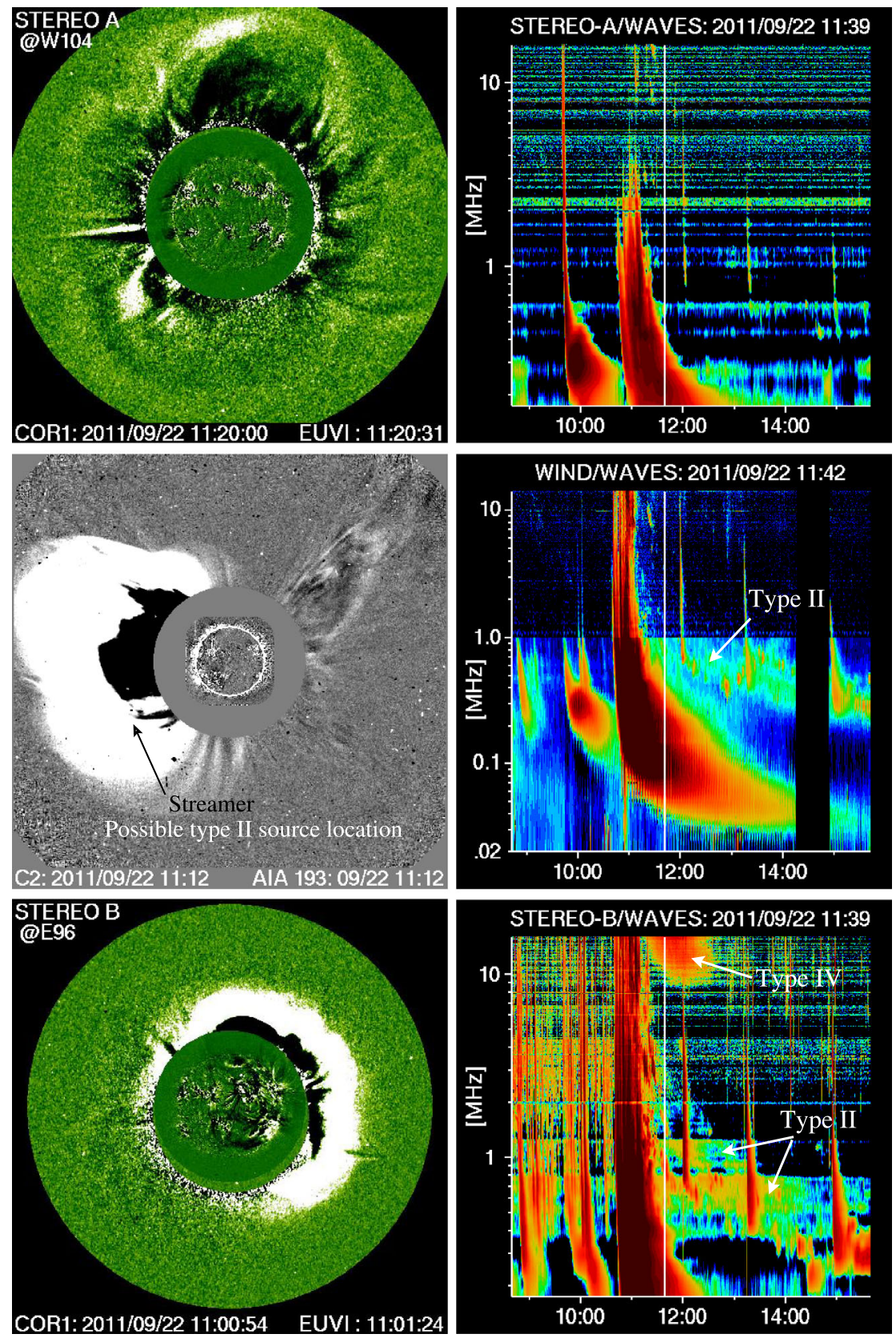

Figure 5 Solar event on 22 September 2011: Flare location at N13E78 in AR 11302.

configuration. The halo CME was first observed by SOHO/LASCO at 22:05 UT, at height $4.35 \mathrm{R}_{\odot}$, with a linear fit speed of $2425 \mathrm{~km} \mathrm{~s}^{-1}$.

An EUV wave that was followed by a dimming originated from the flare location, and it was well observed by STEREO-A. The wave was visible near the limb in the STEREO-B 

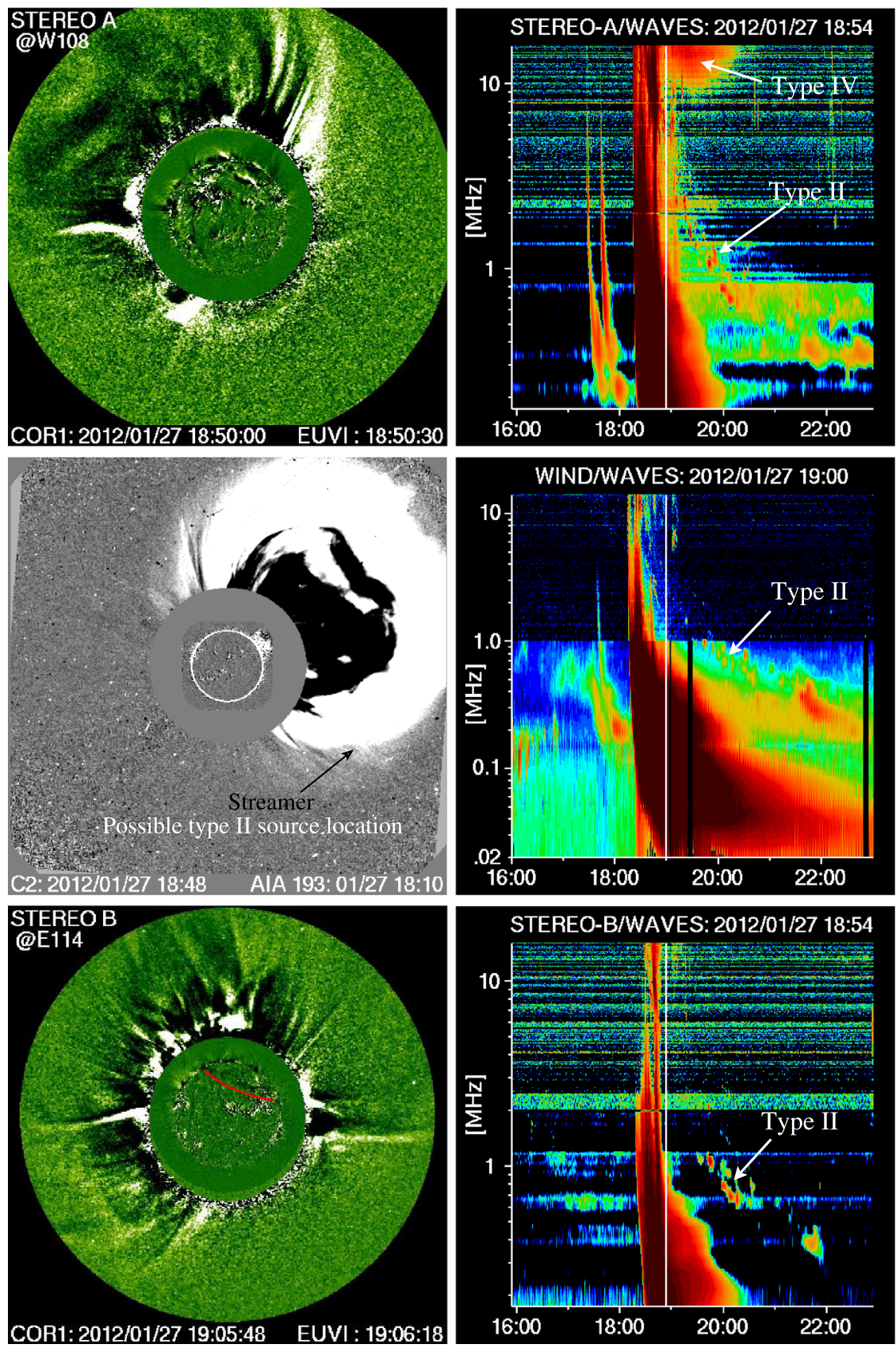

Figure 6 Solar event on 27 January 2012: Flare location at N27W71 in AR 11402. The red line in the STEREO-B EUVI difference image indicates the maximum extent of the EUV wave on the visible disk.

view, but no wave signatures were observed from the Earth view (Figure 4, difference images on the left). 

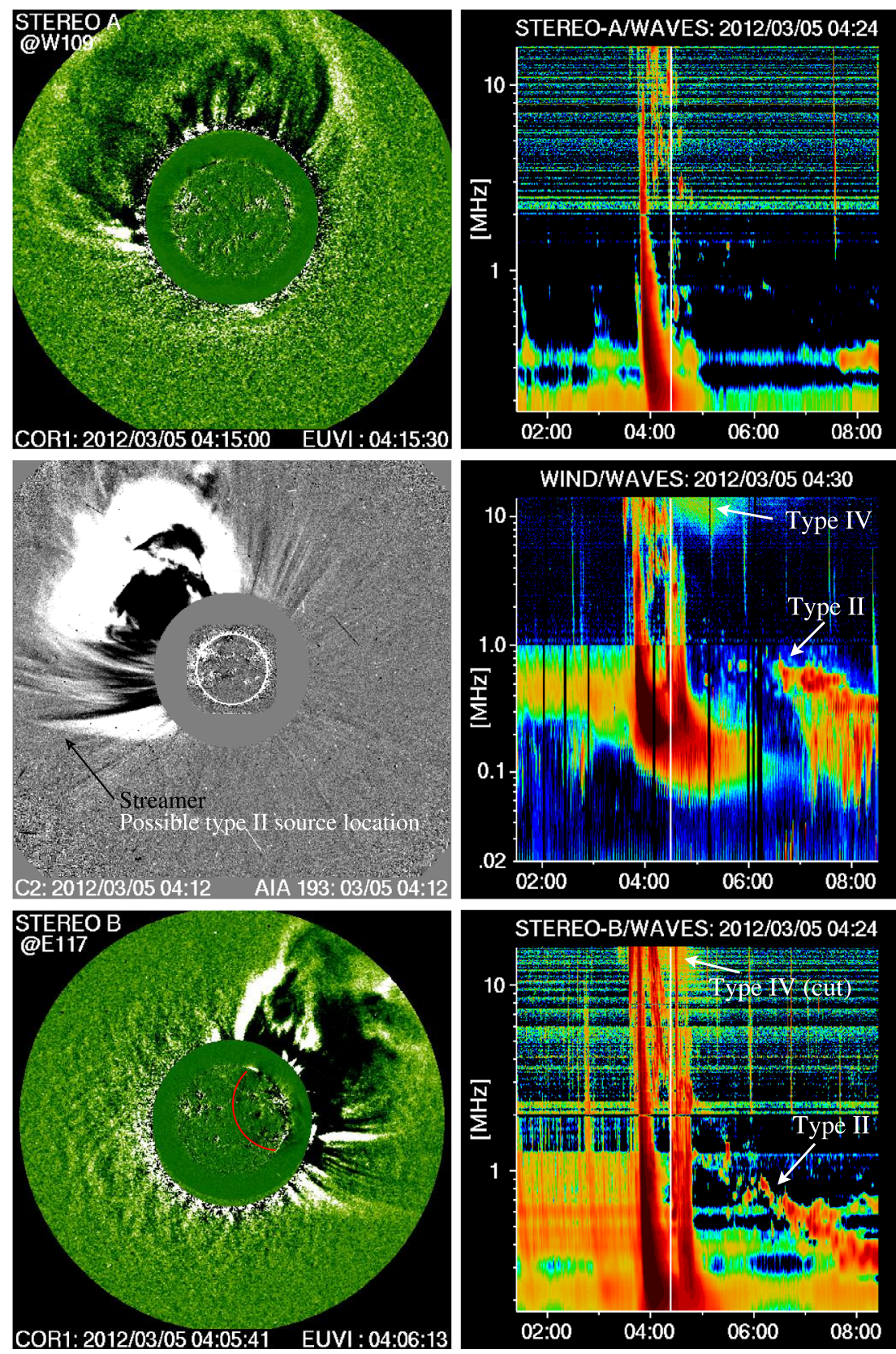

Figure 7 Solar event on 5 March 2012: Flare location at N17E52 in AR 11429. The red line in the STEREO-B EUVI difference image indicates the maximum extent of the EUV wave on the visible disk.

The DH type IV burst was observed to start at 22:15 UT, observed only by STEREO-A, and it reached the lowest frequency of $6 \mathrm{MHz}$ at 23:05 UT. A very fast-drifting type II burst was observed by STEREO-A, but this emission was not detected by the other instruments 
Figure 8 Radio dynamic spectrum observed by STEREO-B/WAVES on 22 September 2011 (bottom) and in the Nançay Decameter Array (NDA) spectrum at $70-10 \mathrm{MHz}$ (top). The flaring region was located at E80 in NDA view and at W10 in STEREO-B view. The metric type IV emission observed by NDA was not detectable in the Wind/WAVES data (same field of view, frequencies below $14 \mathrm{MHz}$ ). The DH type IV burst observed in the STEREO-B field of view does not have a one-to-one match in duration with the metric type IV burst. The type III bursts stop at the type II burst emission lane (indicated by the white dashed line), suggesting that the electron beams cannot pass the type II shock front.
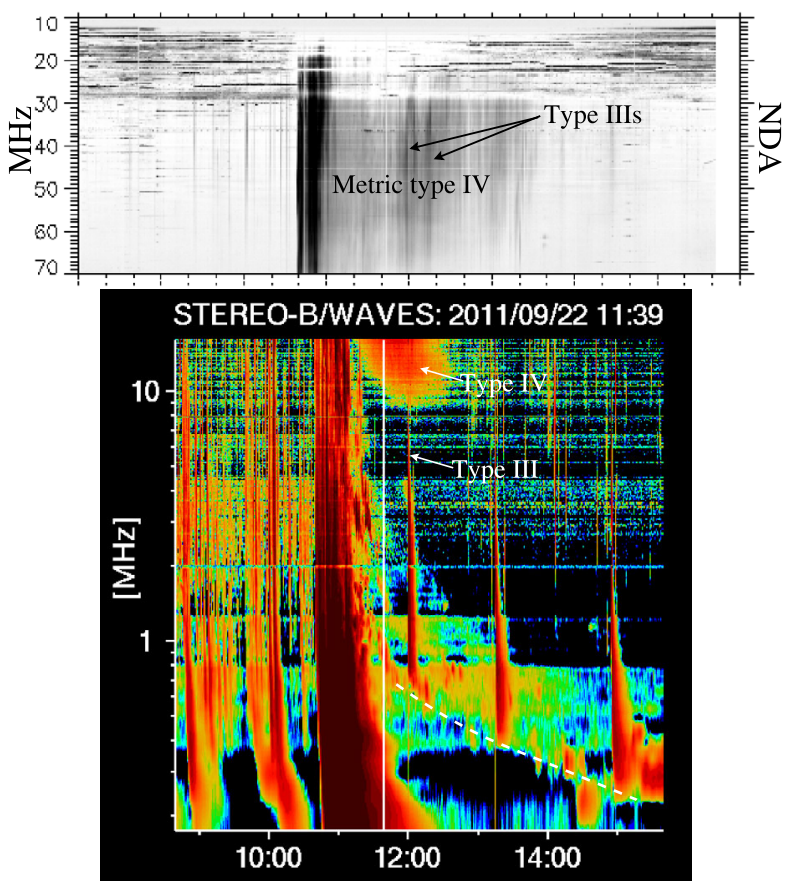

(Figure 4, top right spectrum). The type II burst source is again estimated to be located on the western flank of the CME (Earth view), but this time in a more narrow region that is blocked in view from the other radio instruments.

\subsection{Event on 22 September 2011}

A GOES X1.4 class flare was observed to start at 10:29 UT on 22 September 2011 in Active Region NOAA 11302, located at N13E78. It reached maximum flux at 11:01 UT. A halo CME was first observed by SOHO/LASCO at 10:48 UT, at height $2.98 \mathrm{R}_{\odot}$, and it had a linear fit speed of $1905 \mathrm{~km} \mathrm{~s}^{-1}$. An EUV wave related to this event was mainly observed by STEREO-B/EUVI on the disk (Figure 5, bottom left difference image), but it was also observed to propagate toward the west in Earth view, with a speed of $595 \mathrm{~km} \mathrm{~s}^{-1}$ (Nitta et al., 2013).

The DH type IV burst was observed at 11:10 UT by STEREO-B/WAVES at $16 \mathrm{MHz}$, and it reached the lowest frequency of $8 \mathrm{MHz}$ at 12:15 UT. The DH type IV burst was preceded by a decimetric-metric type IV burst observed by Nançay Decametric Array (NDA) (Earth view). These bursts do not seem to be directly associated, as the metric type IV emission continued after the DH emission had ended, see Figure 8. The drift rates of the emission envelopes do not look to match either. The metric type IV burst shows groups of type III bursts with varying frequency drifts (both positive and negative), similar to those reported by Melnik et al. (2018).

Near 12:00 UT, all the three space instruments recorded a type III burst in their dynamic spectra. The type III burst (formed by a propagating electron beam) is observed to cross the DH type IV burst in the STEREO-B spectrum. This, and also the later type III bursts, stop near the type II emission lane, suggesting that the electron beams cannot pass the type II 
shock front (Figure 8). The location of the type II burst source is most probably on the southeastern flank of the CME (Earth view, Figure 5 middle left difference image), making it observable for STEREO-B and Wind. Figure $2 \mathrm{~b}$ shows some of the open field lines along which electrons could stream out from the active region, and they are mostly directed toward STEREO-B.

\subsection{Event on 27 January 2012}

Active Region NOAA 11402 produced an X1.7 GOES class flare at location N27W71 that started at 17:37 UT and peaked at 18:37 UT on 27 January 2012. The flare was associated with a halo CME that was first observed by SOHO/LASCO at 18:27 UT at height $3.76 \mathrm{R}_{\odot}$, and had a linear fit speed of $2508 \mathrm{~km} \mathrm{~s}^{-1}$. The event was associated with an EUV wave that was observed globally on the disk by STEREO-A/EUVI, and partly on the disk by STEREO-B/EUVI and SDO/AIA. The EUV wave speed from Earth view was determined as $635 \mathrm{~km} \mathrm{~s}^{-1}$ (Nitta et al., 2013).

The DH type IV burst was observed at $16 \mathrm{MHz}$ at 18:30 UT by STEREO-A, but not by the other instruments. The burst reached the lowest frequency of $9 \mathrm{MHz}$ at 19:30 UT. The DH type IV burst was preceded by a metric type IV burst at 18:15 - 18:35 UT, observed by the Green Bank Solar Radio Burst Spectrometer (GBSRBS) down to $300 \mathrm{MHz}$ and by the Radio Solar Telescope Network (RSTN) at 180-25 MHz. Because of the short duration of the metric type IV burst, it is not possible to determine if these two bursts were related.

A type II burst was recorded by all the three space spectrographs, although the type II emission lane was more patchy and narrow in the STEREO-B spectrum (Figure 6). The most probable location for the type II burst source is on the southwestern flank of the CME (Earth view, Figure 6 middle left difference image), thus making it visible in all three fields of view. No type III bursts were observed during the DH type IV burst emission, although some open field lines existed, directed to the northwest (Figure 2c).

\subsection{Event on 5 March 2012}

An X1.1 GOES class flare started at 02:30 UT from the NOAA Active Region 11429 located at N17E52. At 03:12 UT, a filament eruption formed into a CME, first visible in the SOHO/LASCO images at height $3.0 \mathrm{R}_{\odot}$. The linear fit speed of this CME was $594 \mathrm{~km} \mathrm{~s}^{-1}$. A fast halo CME appeared at 04:00 UT at height $4.2 \mathrm{R}_{\odot}$, with a linear fit speed of $1531 \mathrm{~km} \mathrm{~s}^{-1}$, overshadowing the earlier CME. The launch of the second CME was most probably related to the fast rise in X-ray flux, observed to start at 03:25 UT. An EUV wave was observed globally on the disk from Earth view, and it was estimated to move in the southwest direction at a speed of $915 \mathrm{~km} \mathrm{~s}^{-1}$ (Nitta et al., 2013). The wave was also partly observed by STEREO-B/EUVI (Figure 7, bottom left difference image).

The DH type IV burst was observed as a compact event by Wind/WAVES, starting at 04:15 UT and reaching the lowest frequency of $7 \mathrm{MHz}$ at 05:15 UT. It was also observed as shorter-duration ('cut') emission in the STEREO-B view (Figure 7, bottom right dynamic spectrum). This sudden end of emission at all the type IV burst frequencies suggests that the emission was blocked from being observed, rather than ending as an emission process. No significant type III burst activity was observed during the type IV burst emission. Some open field lines are visible in the PFSS maps, however, directed toward the northeast, see Figure 2d.

At decimeter-meter waves, drifting continuum emission was observed at 03:30-05:15 UT (Figure 9, on top, spectrum from NICT/HiRAS). This could be a faint type IV burst or a 
Figure 9 Radio dynamic spectrum observed by Wind/WAVES on 5 March 2012 (bottom) and by HiRAS at 2000-20 MHz (top).
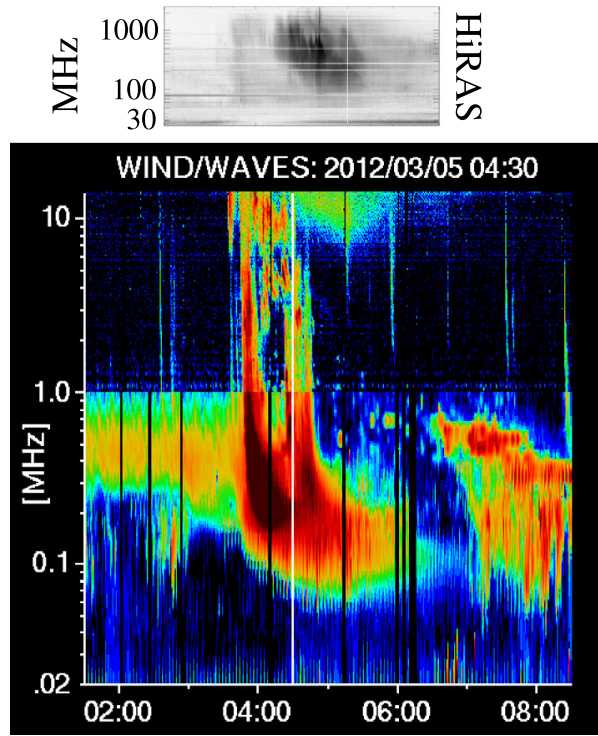

Figure 10 Schematic drawing along the lines presented in Kundu (1965) on where the type IV burst source may be located. Directivity could be related to the extent of the source, which is larger when viewed top-on, in the direction of propagation.

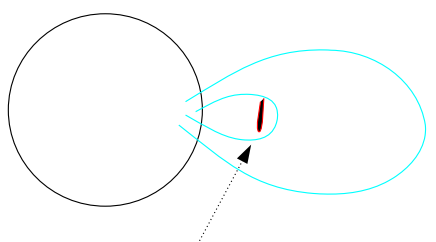

Faint or no type IV emission from this angle

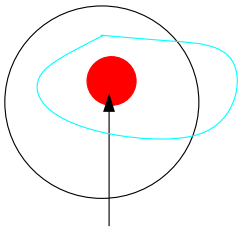

Strong type IV emission when observed top-on

noise storm. The HiRAS spectrum shows that the emission envelope has a drift toward the lower frequencies, with a drift and timing that quite well match those of the DH type IV burst.

The Wind and STEREO-B spectra also show intense type II burst emission. This event has been analyzed by Magdalenić et al. (2014), who used a radio triangulation technique to locate the type II burst source positions. These were found to be close to the southeastern flank of the CME (Earth view, Figure 7, middle left difference image), where streamers were located as well. The appearance of the type II emission was hence suggested to be due to an interaction of shock wave and streamer.

\section{Discussion}

The first ideas to explain why type IV bursts show directivity in their emission were reviewed by Kundu (1965), see especially Fig. $11-12$ in the book and references therein. A schematic drawing in Figure 10 presents the suggested configuration, based on observations of metric type IV bursts as observations from space at low frequencies did not yet exist. A flare explosion ejects a column of gas, and the outward-moving gas carries a magnetic field with it. A small fraction of particles accelerated to very high energies can be trapped in the frozen-in 


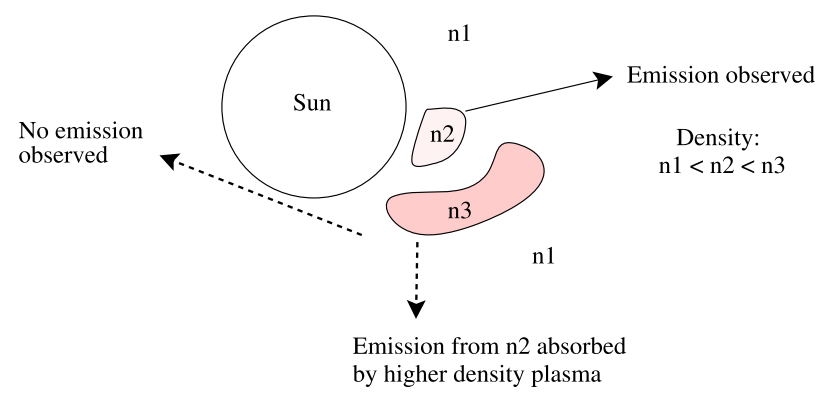

Figure 11 Schematic drawing for not detecting radio emission from a coronal source (n2) that is located behind a region of higher density plasma (n3). Because of the plasma frequency, longer wavelengths produced by plasma emission and/or synchrotron emission in the $\mathrm{n} 2$-region will be stopped and absorbed in the n3-region. In directions not blocked by the n3-region or the Sun itself, the emission can be observed in full.

magnetic fields and will emit continuum (type IV) emission in a wide frequency range. The directivity of emission could then be associated with the large extent of the type IV source in the direction of motion. Later on, a segment of the type IV burst source can separate and move outward, but it will no longer emit synchrotron emission due to the insufficient magnetic fields.

Gopalswamy et al. (2016) recently studied a DH type IV burst on 7 November 2013 that was observed to be intense and complete only in STEREO-B observations, with STEREO-A seeing a partial burst and Wind no burst at all. However, Melnik et al. (2018) reported later that the type IV burst was observed also at decameter waves by URAN-2, from Earth. Gopalswamy et al. (2016) concluded that the type IV emission was directed along a narrow cone, less than $\approx 60$ degrees in width from above the flare site. They suggested that the source of energy for the burst was the flare: electrons accelerated in flare reconnection and then trapped in the post-eruption structures, producing radio emission at the local plasma frequency. As Melnik et al. (2018) were able to observe the start of the type IV burst at decameter waves, they confirmed that the emission was indeed plasma emission since it was highly polarized. Melnik et al. (2018) suggested that the source region for the type IV burst was the CME core, but they did not specify why URAN-2 could observe it while Wind could not.

We note that it is possible that the observed directivity may not be caused by the emission mechanism and/or the extent of the type IV source. Alternatively, the produced radiation could be stopped in certain directions, for example by higher density plasma if it existed in between the source and the observer. In the solar corona, such local density enhancements can occur in coronal streamers and shock-related compression fronts (Kwon and Vourlidas, 2018). Figure 11 shows that emission from the n2-region cannot pass through the higher density plasma in the n3-region, and hence it cannot be observed along this direction. The dense solar atmosphere can also block part of the emission, especially if the source region is located on the far side of the Sun and the direction of propagation is away from the observer.

The five events in our study presented configurations similar as in Figure 11: a CME source region was located in n2-region and a streamer was located in n3-region. Moreover, in all events, a type II burst was estimated to be located near the streamer as a CME flank shock. Therefore, a higher density region existed toward a direction where the type IV burst could not be observed. In the two 4 June 2011 events, the type II - streamer region was on the southwestern CME flank, blocking type IV emission toward the Earth, but being fully observable with STEREO-A. In the 22 September event, the type II-streamer region was 
on the southeastern CME flank, and the type IV burst was observed by STEREO-B, but not by Wind from the Earth direction. In the 27 January 2012 event, the type II-streamer region was on the southwestern CME flank, with a similar configuration as in the 4 June 2011 events. In the 5 March 2012 event, the type II - streamer region was located on the southeastern CME flank (projected Earth view), but the CME propagation direction indicated that the type IV burst source was directed more toward the Earth and the streamer was located behind it. The effect was that the type IV burst was observed by Wind near Earth, but it was only partly visible from STEREO-B.

In the 22 September 2011 event, we observed several type III bursts that ended near the type II burst emission frequency. This type of cutoff has been reported earlier by AlHamadani, Pohjolainen, and Valtonen (2017). One possible explanation for the disappearance of type III radio emission could be a reduced level of beam-driven Langmuir waves, like when two electron beams cross and the radio emission becomes depleted (Briand, Henri, and Hoang, 2014). However, in this scenario the level of radio waves is recovered after the beam crossing, unlike in our event on 22 September 2011. This suggests that the type III bursts could not pass the type II - streamer region either, but were stopped.

\section{Summary and Conclusions}

In this paper, we have studied five solar events that showed intense and compact DH type IV bursts, with a duration of about one hour for most of them, but visible only from one viewing angle. The associated flares had high intensities (three GOES X-class, two unclassified as they originated on the far side of the Sun). All the CMEs had very high speed and were of halo type. Our analysis showed that EUV waves were observed in all of the events, and intense and compact type IV bursts were observed only when the EUV wave propagated globally across the whole visible disk. This may simply indicate the propagation direction of the depleted CME material, if not the directivity of the type IV radio emission.

All the CMEs were associated with type II radio emission at DH wavelengths, which indicates propagating shock fronts driven by CMEs. The calculated estimates for the type II heights showed that the shocks were not bow shocks at the leading fronts of the CMEs, but were located near the CME flanks. In one event, the type II location at the CME flank had also earlier been verified with the radio triangulation technique. All five events included high-density streamers at the deduced locations of the type II shocks. Shock-streamer interaction has been found to be the source of type II burst emission in several earlier studies (e.g., Al-Hamadani, Pohjolainen, and Valtonen, 2017, and references therein). Our analysis showed that in all five events, the type IV emission was not observed from a direction where the type II-streamer region was located in between the CME source region and the observer.

We therefore conclude that a high-density region existed in all events toward the direction where the DH type IV burst could not be observed. As the type II burst sources were found to be located higher in the corona than the type IV sources, the type IV emissions could have been stopped in these shock regions. Dense type II emitting regions could simply be optically thick for the type IV emission. In one of the events, the type II region was also capable of stopping later-accelerated electron beams, visible as type III bursts that ended near the type II burst lane.

To summarize our results and also to tie up our suggested scenario with an earlier analyzed event - where a metric type IV burst could be observed from a direction where the DH type IV burst could not - we present a schematic cartoon in Figure 12. Basically, a type IV 


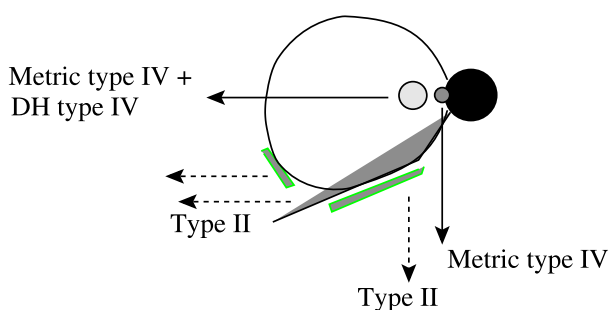

Figure 12 Schematic cartoon that explains why metric type IV emission sources can be observed when DH type IV sources cannot, from the same viewing angle. The green regions indicate possible type II burst locations and the filled gray region shows the streamer position. In this scenario, DH type IV emission is observed only from one viewing angle, because the plasma near the type II emitting region is optically thick for the type IV emission to pass through. The higher density region coincides here with a type II shock-streamer region and it blocks the type IV emission only at longer DH wavelengths, so that the metric type IV emission can still be observed.

burst can be observed from a direction where nothing blocks or absorbs the radio waves. Based on our analysis, possible blocking regions could be located at the CME flanks, where shock-streamer interactions may form type II radio bursts and where electron densities can be much higher than in the surrounding space. These regions may form only higher in the corona, making it possible for the metric type IV bursts to be observable at lower coronal heights. We also note that in most cases, the type II bursts themselves are observed even if the type IV bursts are not.

Acknowledgements We thank the anonymous referee for comments and suggestions that helped to improve this article. We are grateful to all the individuals who have contributed in creating and updating the various solar event catalogs. The CME catalog is generated and maintained at the CDAW Data Center by NASA and the Catholic University of America in cooperation with the Naval Research Laboratory. The Wind WAVES radio type II burst catalog has been prepared by Michael L. Kaiser and is maintained at the Goddard Space Flight Center. SOHO is a project of international cooperation between ESA and NASA. The HMI data are courtesy of NASA/SDO and the HMI science teams. STEREO is part of the NASA Solar Terrestrial Probes (STP) Program. N. Talebpour Sheshvan wishes to thank CIMO (The Centre for International Mobility, Finland) for financial support, contract TM-16-10298.

Disclosure of Potential Conflicts of Interest The authors declare that they have no conflicts of interest.

Open Access This article is distributed under the terms of the Creative Commons Attribution 4.0 International License (http://creativecommons.org/licenses/by/4.0/), which permits unrestricted use, distribution, and reproduction in any medium, provided you give appropriate credit to the original author(s) and the source, provide a link to the Creative Commons license, and indicate if changes were made.

\section{References}

Al-Hamadani, F., Pohjolainen, S., Valtonen, E.: 2017, Solar Phys. 292, 127. DOI.

Bougeret, J.-L., Goetz, K., Kaiser, M.L., Bale, S.D., Kellogg, P.J., Maksimovic, M., et al.: 2008, Space Sci. Rev. 136, 487. DOI.

Bougeret, J.-L., Kaiser, M.L., Kellogg, P.J., Manning, R., Goetz, K., Monson, S.J., Monge, N., Friel, L., Meetre, C.A., Perche, C., Sitruk, L., Hoang, S.: 1995, Space Sci. Rev. 71, 231. DOI.

Briand, C., Henri, P., Hoang, S.: 2014, J. Geophys. Res. 119, 2365. DOI.

Cane, H.V.: 1984, Astron. Astrophys. 140, 205.

Cunha-Silva, R.D., Fernandes, F.C.R., Selhorst, C.L.: 2015, Astron. Astrophys. 578, A38. DOI.

Gopalswamy, N., Akiyama, S., Mäkelä, P., Yashiro, S., Cairns, I.H.: 2016, In: URSI Asia-Pacific Radio Science Conference in Seoul, August 21-25, 2006. arXiv. 
Hillaris, A., Bouratzis, C., Nindos, A.: 2016, Solar Phys. 291, 2049. DOI.

Krupar, V., Maksimovic, M., Kontar, E.P., Zaslavsky, A., Santolik, O., Soucek, J., Kruparova, O., Eastwood, J.P., Szabo, A.: 2018, Astrophys. J. 857, 82. DOI.

Kundu, M.R.: 1965, Solar Radio Astronomy, Interscience, New York.

Kwon, R-Y., Vourlidas, A.: 2017, Astrophys. J. 836, 246. DOI.

Kwon, R-Y., Vourlidas, A.: 2018, J. Space Weather Space Clim. 8, A08. DOI.

Leblanc, Y., Dulk, G.A., Vourlidas, A., Bougeret, J.-L.: 2001, J. Geophys. Res. 106, 25301. DOI.

Melnik, V.N., Brazhenko, A.I., Konovalenko, A.A., Dorovskyy, V.V., Rucker, H.O., Panchenko, M., Frantsuzenko, A.V., Shevchuk, M.V.: 2018, Solar Phys. 293, 53. DOI.

Magdalenić, J., Marqué, C., Krupar, V., Mierla, M., Zhukov, A.N., Rodriguez, L., Maksimović, M., Cecconi, B.: 2014, Astrophys. J. 791, 115. DOI.

Nindos, A., Aurass, H., Klein, K.-L., Trottet, G.: 2008, Solar Phys. 253, 3. DOI.

Nitta, N.V., Schrijver, C.J., Title, A.M., Liu, W.: 2013, Astrophys. J. 776, 58. DOI.

Pick, M., Vilmer, N.: 2008, Astron. Astrophys. Rev. 16, 1. DOI.

Vršnak, B., Magdalenič, J., Zlobec, P.: 2004, Astron. Astrophys. 413, 753. DOI. 